\title{
Optimizing the Specific Removal Rate with the Burst Mode Under Varying Conditions
}

\author{
Beat Jaeggi ${ }^{* 1}$, Stefan Remund ${ }^{* 1}$, Yiming Zhang*1 ${ }^{*}$ Thorsten Kramer $^{* 1}$ and Beat Neuenschwander ${ }^{* 1}$ \\ ${ }^{* 1}$ Bern University of Applied Sciences, Institute of Applied Laser, Photonics and Surface Technolo- \\ gies, Pestalozzistrasse 20, CH-3400 Burgdorf, Switzerland
}

E-mail: beat.jaeggi@bfh.ch

\begin{abstract}
The influence of pulse-bursts is investigated using different spot sizes and different wavelengths as well. The specific removal is calculated and compared as a function the peak fluence of the single pulses (not total fluence of the burst package). A drop in the specific removal for copper and aluminum is observed for a 2 pulse-burst, whereas for a 3 pulse-burst the specific removal rate is larger than for the 2 pulse-burst and may exceed the rate observed in the single pulse mode in special cases. Possible explanations for these observations are given based on shielding effects and a preconditioning by previous pulses. The usage of pulse-bursts may also increase the process window and in addition influence the surface quality. The formation of small cavities on the machined surface often appearing in steel can be shifted to higher fluences if pulse-bursts are applied.
\end{abstract}

DOI: 10.2961/jlmn.2017.03.0015

Keywords: burst mode, pulse-burst, ps-pulses, specific removal rate, energy efficiency

\section{Introduction}

Ultrashort laser pulses are used if high requirements regarding quality and precision are demanded. These USP laser systems have shown their applicability on different materials like metals, semiconductors and insulators in manifold applications. For industrial applications the demand for high throughput regarding the utilization of ultrafast laser radiation is not yet met and increasing productivity and efficiency still represents a key factor in today's research. Even if the maximum average power of industrial ultrashort pulsed systems has reached the $100 \mathrm{~W}$ range [1], and the $\mathrm{kW}$-range for scientific systems [2,3], the use of such high powers is still a challenge. There exist some single applications where high pulse energies are needed, for example the cutting of CFRP [4]. For machining metals, mostly moderate peak fluences in the range of a couple $\mathrm{J} / \mathrm{cm}^{2}$ are required to work at the optimum point, where the ablation process is most energy efficient [5,6]. Applying too high pulse energies leads to poor quality and an inefficient process. Therefore the full average power of today's laser systems can only be applied by either increasing the spot size to remain in the right fluence level or by increasing the repetition rate, scan speed and average power to preserve the peak fluence and the spatial pulse to pulse distance. For common galvanometric scanners the maximum scan speed is in the range of $10 \mathrm{~m} / \mathrm{s}$ using a standard $160 \mathrm{~mm}$ focusing objective. Higher deflection speeds can be reached by using polygon line scanners [7], by machining rotating cylinders or drums $[8,9]$ or by using AOM or EOM scanners [10]. Another way is to split the single output beam in several beams by using DOE's [11] or SLM technology [12] thus a kind of energy sharing.

Ultrashort pulsed laser systems are mostly built in a MOPA (Master-Oscillator-Power-Amplifier) arrangement, where the seed oscillator operating at the frequency $f_{s}$ is followed by a pulse picker to reduce the repetition rate down to the desired output frequency (repetition rate) of the laser system $f_{L}$. This pulse train is then amplified in the subsequent amplifier stages. The pulse picker is capable of letting pass not only single pulses but a sequence of $n$ pulses which are then amplified. The group of $n$ pulses is then called a burst. The time spacing between two bursts is given by $\Delta t_{L}=1 / f_{L}$, while the temporal spacing between the single pulses in the burst is equal to $\Delta t_{B}=1 / f_{s}$. If there is not only a pulse picker but also an attenuator between seedoscillator and amplifier stages, the shape of the burst, meaning the energy distribution in the burst can be varied. It is therefore possible to generate a burst in which each pulse has the same pulse energy. In addition the temporal spacing, i.e. the time distance between two subsequent pulses in the burst may be varied in multiples of the spacing of the seed oscillator.

Bursts are used for different application e.g. in glass processing for welding [13], writing of gratings or waveguides [14] or cutting with different technologies [15-18]. Also for machining metals several publications deal with pulse-bursts. In 2010 pulse-bursts have been reported to be more efficient than single pulses as e.g. an about 10 times higher removal rate for stainless steel could be achieved by using a 10 pulse-burst compared to single pulses at the same repetition rate and average power [19]. However, the main part of this effect can be explained by the reduced energy of the individual pulses in the burst, which is nearer the optimum value going with highest efficiency [20]. Nevertheless it has also been reported that the burst mode has an influence onto the ablation process itself. In [21] it is shown that a 2 pulse-burst with a total energy of $22 \mu \mathrm{J}$ is about $10 \%$ more efficient than twice a single pulse with a pulse energy of $11 \mu \mathrm{J}$ on steel C75 using 12 ps pulses. In contrast a total energy of $4 \mu \mathrm{J}$ is not more efficient than twice a single pulse with $2 \mu \mathrm{J}$. In addition it has been shown that for copper, silver and gold a 2 pulse-burst leads 
to a specific removal rate which is reduced by more than $50 \%$ implying that the second pulse is even re-depositing material [22]. In contrast, the third pulse in a 3 pulse-burst is then twice to three times as efficient as a single pulse. This results in a maximum specific removal rate which is identical to the one of single pulses in the case of silver and gold or which is even $15 \%$ higher in the case of copper [22]. As shown in [20] and [23] the time spacing between the pulses in the burst as well as the energy distribution inside the burst may have an influence on the specific removal rate, meaning on the ablated volume per time and average power.

In [24] the influence of the ablation cooling effect is discussed. This effect takes benefit from the ablation of the target material before the residual heat deposited by previous pulses diffuses away from the processing region i.e. less energy is finally heating up the material which leads to higher removal rates. But these experiments (whenever they show really higher specific removal rates) were performed at very high repetition rates in the $\mathrm{GHz}$ regime corresponding to a temporal spacing between the single pulses in the burst of $1 \mathrm{~ns}$ and less as well as with a high number of pulses in a burst sequence. The conditions in the experiments presented in [19][20][21][22][23] and also in the following are completely different. The number of pulses in a burst is limited to a maximum of 10 and the temporal spacing between the single pulses in the burst amounts 12 ns ore more which is at least one magnitude higher. Ablation cooling is therefore assumed to have no significant influence onto the experimental results presented here.

In addition, in [25][26] an influence of the spot size on the specific removal rate is shown. An influence of the spot size on the ablation threshold is shown in [27]. We will not investigate the ablation threshold here, but [27] may also indicate an influence of the spot size on the ablation process. In this work we investigate the influence of the spot size, the wavelength and especially for copper the influence of a 5 pulse-burst on the specific removal rate.

\section{Experimental setup}

For the experiments a FUEGO ps-laser system from Lumentum (former Time-Bandwidth) is used. To check the influence of the wavelength the fundamental wavelength of $1064 \mathrm{~nm}$ and the SHG of $532 \mathrm{~nm}$ are used. The repetition rate in principle can be varied between $200 \mathrm{kHz}$ and $8 \mathrm{MHz}$, but is fixed to $200 \mathrm{kHz}$, where the highest pulse energies are obtained. The frequency of the seed-oscillator amounts $82 \mathrm{MHz}$ which leads to a pulse separation in the burst of 12 ns. Via the FlexBurst ${ }^{\mathrm{TM}}$ option a burst up to 8 pulses with a spacing of 12 ns can be generated whereas the energy of each pulse in the burst can be adjusted individually.

The laser beam is guided via several folding mirrors to the beam expander, placed in front of the galvo scanner. To achieve circular polarization, a $\lambda / 4$-waveplate is introduced in the beam path. The used galvo scanner is an intelliSCAN $N_{\text {se }} 14$ from SCANLAB. To achieve different spot sizes different focusing objectives with $160 \mathrm{~mm}$ and $100 \mathrm{~mm}$ focal length are used and the corresponding beams were measured with a BP104-UV beam profiler from Thorlabs at the repetition rate of $1 \mathrm{MHz}$. Table 1 shows the resulting spot radii $w_{0}$ in the focal plane, the beam quality $M^{2}$ for the used objectives and the different wavelengths.
Table 1 Spot size, beam quality and size of the machined square for the different objectives and wavelength

\begin{tabular}{ccccr}
\hline$\lambda / \mathrm{nm}$ & $f_{o b j} / \mathrm{mm}$ & $w_{0} / \mu \mathrm{m}$ & \multicolumn{1}{c}{$M^{2}$} & \multicolumn{1}{c}{$s / \mu \mathrm{m}$} \\
\hline 1064 & 100 & 11.7 & $\leq 1.7$ & 1 1'111.5 \\
1064 & 160 & 15.5 & $\leq 1.36$ & $1^{\prime} 472.5$ \\
532 & 100 & 4.4 & $\leq 1.13$ & 990.0 \\
532 & 160 & 7.2 & $\leq 1.15$ & $1{ }^{\prime} 494.0$ \\
\hline
\end{tabular}

The galvo scanner is controlled by the own developed synchronization solution [28][29]. The pitch meaning the pulse to pulse and the line to line distance was set to $w_{0} / 2$ corresponding to an overlap $75 \%$ with respect to the spot size. To get the specific removal rates, squares with a given side length $s$ are machined with different average powers and their depth $z$ is measured using the white light interferometric microscope smartWLI from GBS. Together with the used repetition frequency $f_{L}$, the average power $P_{a v}$, the number of machined layers $N_{l}$ and the pitch $p$ the specific removal rate can be calculated according to [20].

$$
d V / d t \cdot P_{a v}=d V / d E=\frac{z \cdot p^{2}}{N_{l}} \cdot \frac{f_{L}}{P_{a v}}
$$

The specific removal rate is defined as the removed material per laser-on-time and applied average power. For an industrial application the duty cycle of the machine has to be taken in account as well, to calculate the time needed to machine a structure. Different sample materials like copper Cu-DHP (in US: Cu 12-200), stainless steel 1.4301 (in US: AISI 304) and aluminum Al99.5 (in US: 1050A) are used. Different numbers of layers are used to machine the squares for the different materials, wavelength and pulses in the burst. The peak fluence of the single pulses in the burst $\phi_{0, s}$ is kept equal to the single pulse operation. This means for a 2 pulse-burst the pulse energy for each pulse in the burst is equal to the pulse energy for the single pulse, i.e. the average power is doubled compared to the single pulse. The total number of pulses applied per structure is hold constant. Therefore the number of layers is reduced for the burst mode. Table 2-4 show the corresponding number of layers for the different wavelengths and materials.

Table 2 Number of layers $N_{l}$ for copper

\begin{tabular}{ccc}
\hline Pulses in the Burst & $\lambda=1064 \mathrm{~nm}$ & $\lambda=532 \mathrm{~nm}$ \\
\hline 1 & 48 & 48 \\
2 & 24 & 24 \\
3 & 16 & 16 \\
4 & 12 & - \\
5 & 10 & - \\
\hline
\end{tabular}

After measuring the ablation depth, the calculated specific removal rate is plotted as a function of the peak fluence of a single pulse (not the total fluence in the burst). 
Table 3 Number of layers $N_{l}$ for stainless steel

\begin{tabular}{ccc}
\hline Pulses in the Burst & $\lambda=1064 \mathrm{~nm}$ & $\lambda=532 \mathrm{~nm}$ \\
\hline 1 & - & 96 \\
2 & - & 48 \\
3 & - & 32 \\
4 & - & 24 \\
5 & - & 19 \\
\hline
\end{tabular}

Table 4 Number of layers $N_{l}$ for aluminum

\begin{tabular}{ccc}
\hline Pulses in the Burst & $\lambda=1064 \mathrm{~nm}$ & $\lambda=532 \mathrm{~nm}$ \\
\hline 1 & 96 & 48 \\
2 & 48 & 24 \\
3 & 32 & 16 \\
\hline
\end{tabular}

From all investigated materials steel 1.4301 shows the lowest thermal diffusivity and therefore heat accumulation and scan strategies should first have an influence on the results for steel. But in [20] it was shown that

- no change in the specific removal rate was observed for steel in similar configuration with a pitch of $w_{0} / 2$ up to a repetition rate of $1.6 \mathrm{MHz}$

- the estimated temperature just before the next pulse strikes on the surface for a n-pulse burst is almost equal to the one of a single pulse with $n$ times higher energy

As the experiments presented here are all performed at a laser repetition rate of $200 \mathrm{kHz}$ heat accumulation and therefore also scanning strategies are assumed to have no influence onto the measured specific removal rate.

\section{Experimental results}

\subsection{Results for copper}

As shown in previous publications [22,23], a 3 pulseburst will lead to a higher specific removal rate compared to a single pulse operation and a 2 or 4 pulse-burst.

This study was repeated in a chamber at vacuum condition and also using different shielding gases. Neither the vacuum condition nor the shielding gas has an influence on less efficient 2 pulse-burst or the more efficient 3 pulseburst. The absolute values of the measured specific removal rate are lower compared to that one's machined in air, due to the fact the no suction system could be used in the vacuum chamber.

In addition the study in air was repeated to check the influence of the 5 pulse-burst. The obtained result is shown in Fig. 1.

Comparing the maximum specific removal rate, the 2 pulse-burst shows an about $60 \%$ smaller rate compared to the single pulse. In contrast, using a 3 pulse-burst the maximum rate is increased by about $13 \%$ compared to single pulses. The 4 pulse-burst shows again an about $25 \%$ smaller maximum value compared to the single pulse, and the peak fluence where the maximum specific removal rate is obtained is shifted to higher fluence values. Using a 5 pulse-burst will lead to a similar effect as a three pulse- burst. The more efficient material removal using a 5 pulseburst was also reported in [30] for percussion drilling of microholes using a 10 ps laser and a time spacing of 20 ns between the pulses in the burst.

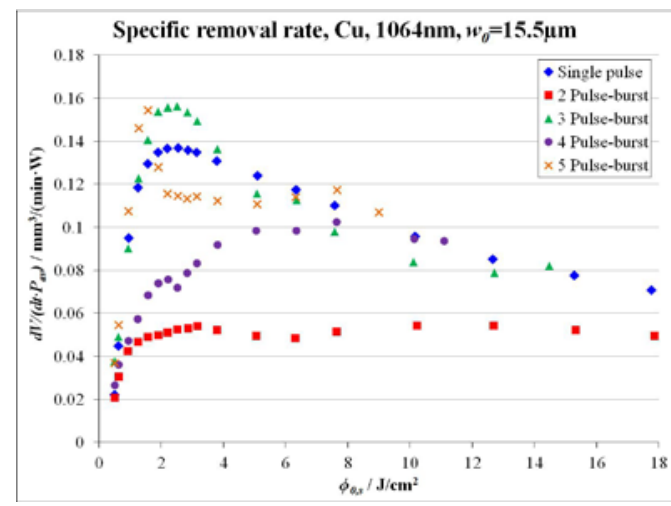

Fig. 1. Specific removal rate for copper, $\lambda=1064 \mathrm{~nm}, w_{0}=15.5 \mu \mathrm{m}$

In addition a second focal objective with a smaller focal length was used to check the influence of the spot radius in case of the IR wavelength. The results are summarized in Fig. 2.

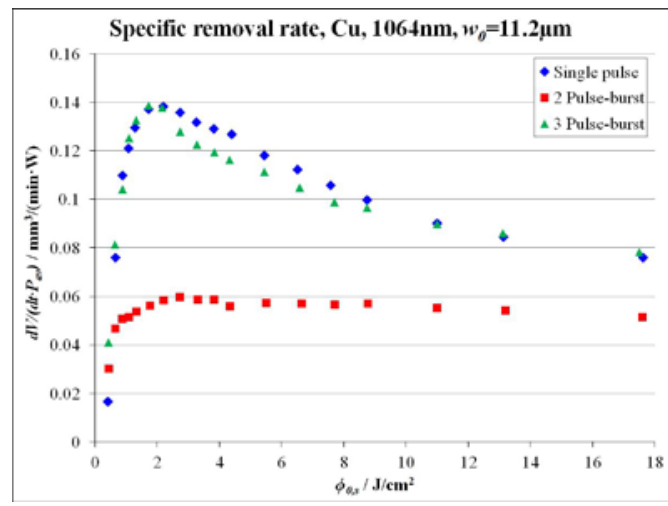

Fig. 2. Specific removal rate for copper, $\lambda=1064 \mathrm{~nm}, w_{0}=11.2 \mu \mathrm{m}$

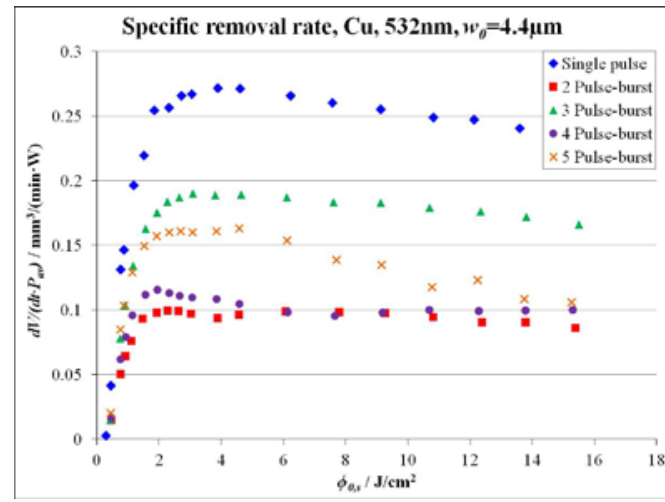

Fig. 3. Specific removal rate for copper, $\lambda=532 \mathrm{~nm}$, $w_{0}=4.4 \mu \mathrm{m}$

The ablation behavior for a single pulse is not influenced by the spot size. Also the maximum rate for a 2 pulse-burst is almost equal and amounts about $55 \%$ of the maximum rate observed for single pulses. But the 3 pulseburst is no longer more efficient than a single pulse.

The change of the wavelength to $532 \mathrm{~nm}$ leads to an again smaller spot size. The specific removal rate for a single pulse is increased, compared to the IR, by a factor of 
two (see Fig. 3). Again the 2 pulse-burst shows a maximum value which is less than $50 \%$ compared to the single pulse.

\subsection{Results for stainless steel}

Machining of stainless steel with $1064 \mathrm{~nm}$ wavelength and a spot radius of $16 \mu \mathrm{m}$ will lead to the measurements shown in Fig. 4 which were published in [20].

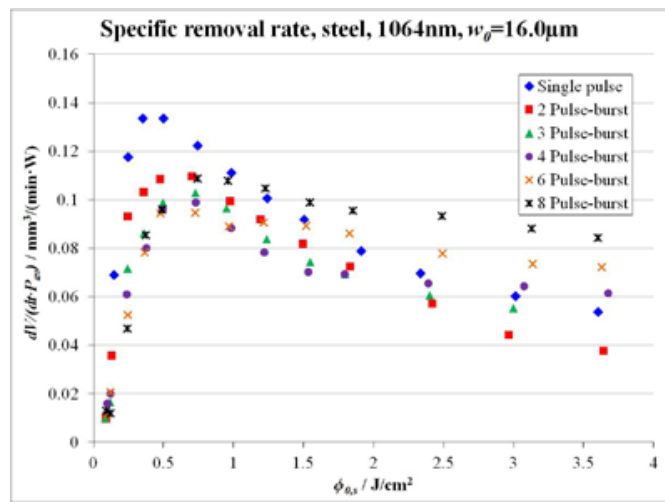

Fig. 4. Specific removal rate for stainless steel, $\lambda=1064 \mathrm{~nm}$, $w_{0}=16.0 \mu \mathrm{m}$

For a comparison also this material was machined at $532 \mathrm{~nm}$ wavelength and a spot radius of $7.2 \mu \mathrm{m}$. Compared to the observed values in [20], machining with $532 \mathrm{~nm}$ $\left(w_{0}=7.2 \mu \mathrm{m}\right)$ and single pulses lead to an increase of the specific removal rate of about $15 \%$ (see Fig. ). In addition the drop in specific removal rate from single pulse operation two a 2 pulse-burst is larger for the visible wavelength.

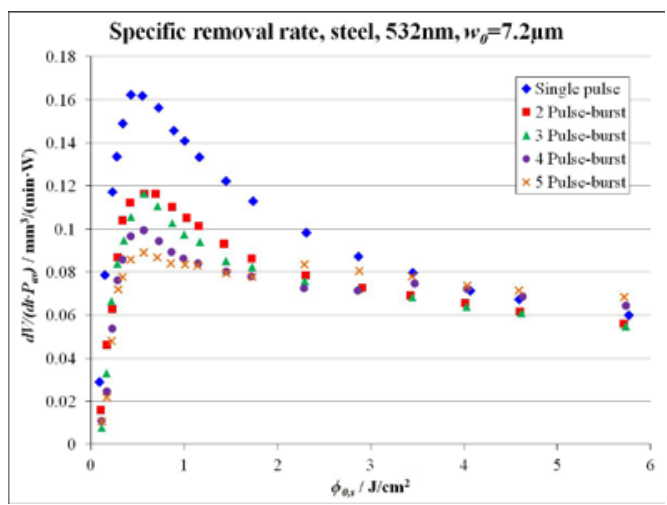

Fig. .: Specific removal rate for stainless steel, $\lambda=532 \mathrm{~nm}$, $w_{0}=7.2 \mu \mathrm{m}$

Machining with single pulses at a too high peak fluence will lead to a strong formation of cavities and spikes, which have to be avoided for most applications. [31] describes the laser joining of a polymer on a stainless steel surfaces, where the formation of these cavities and spikes are helpful to get a higher specific adhesion. This crater formation observed at high fluences can be suppressed or shifted to higher fluences if pulse-bursts are used, as shown in Fig. 5 and also observed in [34].

\subsection{Results for aluminum}

If aluminum is treated with $1064 \mathrm{~nm}$ wavelength and a spot radius of $15.5 \mu \mathrm{m}$ a similar behavior as for copper can be observed as shown Fig. 6.
The maximum specific removal rate for a 2 pulse-burst is about $53 \%$ smaller compared to the maximum observed for single pulses and the 3 pulse-burst shows an increase of the maximum specific removal rate of about $13 \%$. The location of the maximum specific removal rate depends on the number of pulses in the burst.

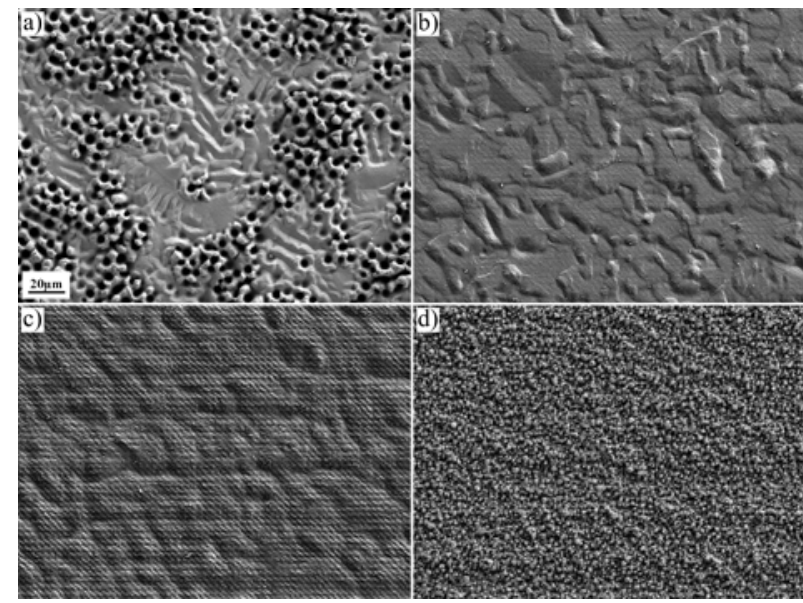

Fig. 5. SEM images (SE2 detector) of the surface quality for stainless steel, $\lambda=532 \mathrm{~nm}, w_{0}=7.2 \mu \mathrm{m}, \phi_{0, s}=5.7 \mathrm{~J} / \mathrm{cm}^{2}$; a) Single pulse; b) 2 pulse-burst; c) 3 pulse-burst; d) 5 pulse-burst

In contrast, for $532 \mathrm{~nm}$ wavelength the usage of the burst mode will not lead to an increase of the specific removal rate (see Fig. 7).

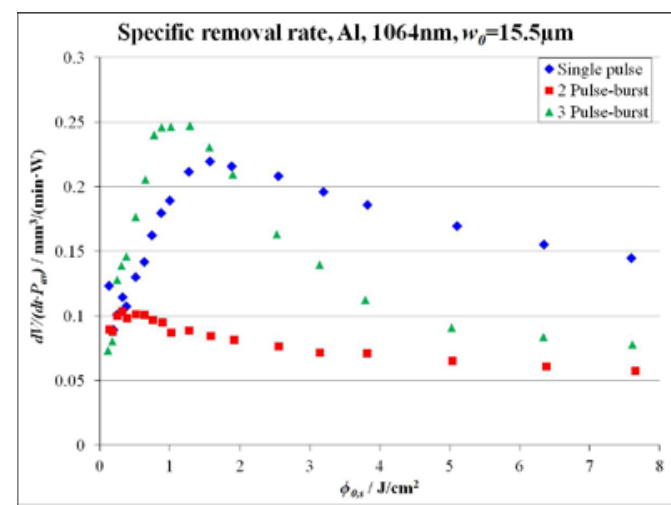

Fig. 6. Specific removal rate for aluminum, $\lambda=1064 \mathrm{~nm}$, $w_{0}=15.5 \mu \mathrm{m}$

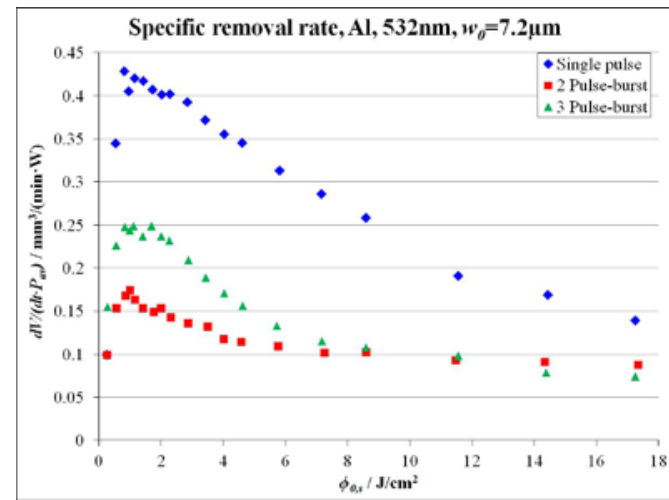

Fig. 7. Specific removal rate for aluminum, $\lambda=532 \mathrm{~nm}$, $w_{0}=7.2 \mu \mathrm{m}$ 
Both, the 2 pulse-burst and the 3 pulse-burst show maximum specific removal rates which are lower than the maximum rate observed with single pulses. The reduction amounts about $60 \%$ and $42 \%$ respectively. For the single pulse operation, the maximum specific removal rate for $532 \mathrm{~nm}$ is about factor two higher compared to $1064 \mathrm{~nm}$ wavelength. The maximum specific removal rate for a 2 pulse-burst is for both wavelengths more than 50\% smaller as for the single pulse.

\section{Discussion}

\subsection{2 pulse-burst}

For a 2 pulse-burst the maximum specific removal rate is much smaller for copper and aluminum, independent of the used wavelength and is always smaller than $50 \%$ of the maximum rate observed with single pulses. This decrease can be addressed to a shielding effect. The second pulse is absorbed, reflected or scattered in the particle plume of the first pulse. The ablated volume per pulse for a Gaussian beam can be calculated and amounts [6]:

$$
d V_{a b l}=\frac{1}{4} \cdot \pi \cdot w_{0}^{2} \cdot \delta \cdot \ln ^{2}\left(\frac{\phi_{0}}{\phi_{t h}}\right)
$$

With $\delta$ as the energy penetration depth, $\phi_{0}$ the used laser peak fluence and $\phi_{t h}$ the threshold fluence of the sample material.

The authors in [32] assume that the plume is expanding with a fixed aperture angle, which depends on the used laser fluence and is independent of the spot diameter. In [33] it is shown, that the angle spread of the particle plume is small in the first tens of ns after the impact of the laser pulse. For simplicity we assume therefore that the plume is cylindrically shaped in the first phase of the expansion close to the sample surface. Therefore the volume of the particle plume can be written as:

$$
d V_{p l}=\pi \cdot w_{0}^{2} \cdot h
$$

Where $h$ denotes the height of the plume which depends on the propagation speed of the particles $v$ and the time between two pulses $\Delta t_{B}$ :

$$
h=v \cdot \Delta t_{B}
$$

Assuming that the ablated material is complete transformed in to particles, the particle density in the plume is then proportional to the relation of the energy penetration depth, the propagation speed of the plume and the pulse separation:

$$
\rho_{p l}=\frac{d V_{a b l}}{d V_{p l}} \propto \frac{\delta}{v \cdot \Delta t_{B}}
$$

The higher the energy penetration depth, the more material is ablated per pulse, and the higher is the particle density in the particle plume. The smaller the pulse separation, the higher is the density of the particle plume. For the materials copper and aluminum, the energy penetration depth is quite high, and therefore the second pulse is shielded more due to the more dense particle plume, compared to stainless steel for the pulse separation of 12 ns. If the pulse separation is short enough, also for steel a strong shielding, i.e. a strong decrease of the specific removal rate is observed for a 2 pulse-burst [21][34]. If the individual pulses in a 2 pulse-burst are separated only several tens of picoseconds, plasma shielding will play a roll, as shown in [35][36]. In [20] is shown, that an increase of the time spacing of the two pulses will lead to a recovery of the specific removal rate to the values observed with single pulses. This recovery will take place for a spacing of 24 ns in the case of stainless steel where for copper still at a spacing of $60 \mathrm{~ns}$ a drop of about $40 \%$ is observed. If the second pulse in the burst is completely absorbed, reflected or scattered by the particle plume, the specific removal rate should not fall below the half of the one observed for a single pulse. As a drop of the specific removal rate of more than $50 \%$ is observed for copper and aluminum, the second pulse in the burst will therefore also affect the following pulses. Also shock waves are often discussed as e.g in [37]. But there the used fluences were one or more magnitudes higher. Shock waves are therefore assumed to play only a minor role for the experiments presented here.

If the specific removal rates $d V /\left.d E\right|_{1}, \ldots . . d V /\left.d E\right|_{n}$ were measured for a sequence of bursts, starting from single pulses up to $n$ pulses per burst, the relative contribution $\eta_{\text {rel,k }}$ or normalized differential rate of each pulse can be calculated as follows:

$$
\eta_{r e l, k}=\frac{k \cdot d V /\left.d E\right|_{k}-(k-1) \cdot d V /\left.d E\right|_{k-1}}{\max \left(d V /\left.d E\right|_{1}\right)}
$$

This calculation is done under the assumption that for a $n$-pulse burst the specific removal rate of the first $n-1$ pulses equals the one of a $n-1$ pulse burst.

The result for copper, machined in the IR and green is shown in Fig. 8 and Fig. 10, respectively. The $2^{\text {nd }}$ and also the $4^{\text {th }}$ pulse in the burst re-deposit material on the surface because of the negative contribution to the ablation process what is equal to a drop in the specific removal rate larger than $50 \%$.

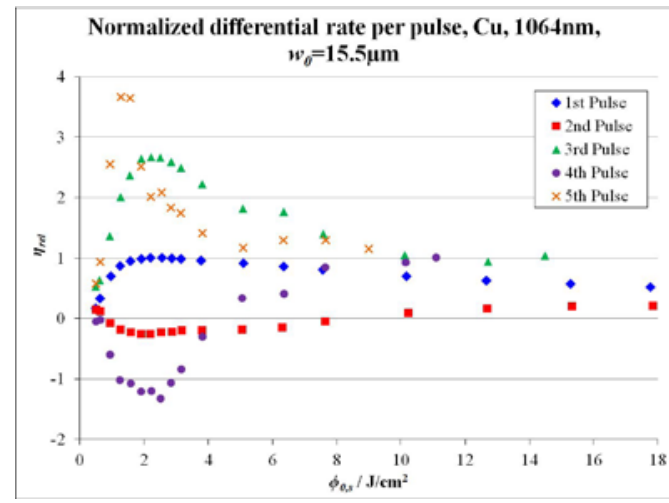

Fig. 8. Normalized differential rate per pulse for copper, $\lambda=1064 \mathrm{~nm}, w_{0}=15.5 \mu \mathrm{m}$

In [38] it is assumed that the absorption of the second pulse in the particle plume creates enough momentum that some parts of the nascent plume produced by the first pulse are driven back on the surface. 


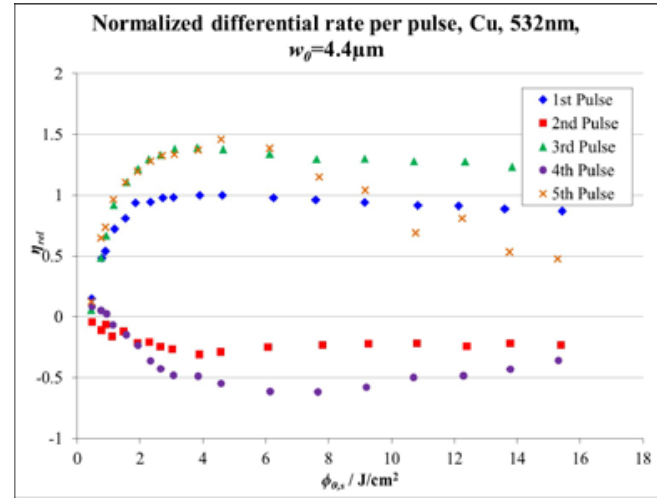

Fig. 9. Normalized differential rate per pulse for copper, $\lambda=532 \mathrm{~nm}, w_{0}=4.4 \mu \mathrm{m}$

For copper machined with $1064 \mathrm{~nm}$ this effect is confirmed by SEM images, where re-deposited particles are clearly visible as melted plates, shown in Fig. 10.

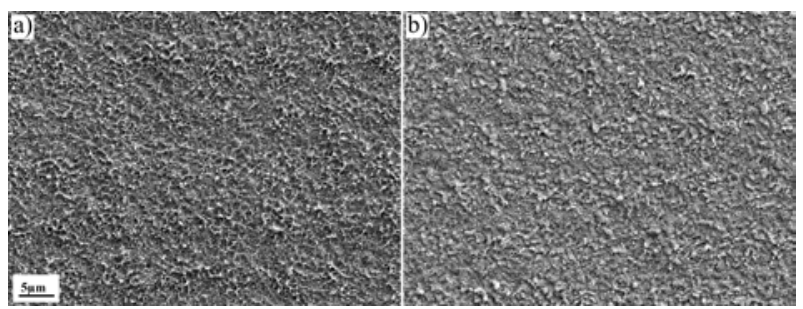

Fig. 10. SEM images (SE2 detector) of the surface quality for copper, $\lambda=1064 \mathrm{~nm}, w_{0}=15.5 \mu \mathrm{m}, \phi_{0, s}=2.2 \mathrm{~J} / \mathrm{cm}^{2}$; a) Single pulse; b) 2 pulse-burst

The change of the wavelength to $532 \mathrm{~nm}$ results in a different surface. A re-deposition of material can no longer be confirmed by the SEM images as shown in Fig. 11. The surface for the 2 pulse-burst no longer shows large melt splashes or plates. But it seems that the surface is less smooth compared to the single pulse and shows much more small melting droplets. In general the surfaces produced with $532 \mathrm{~nm}$ show less melting worms on the surface.
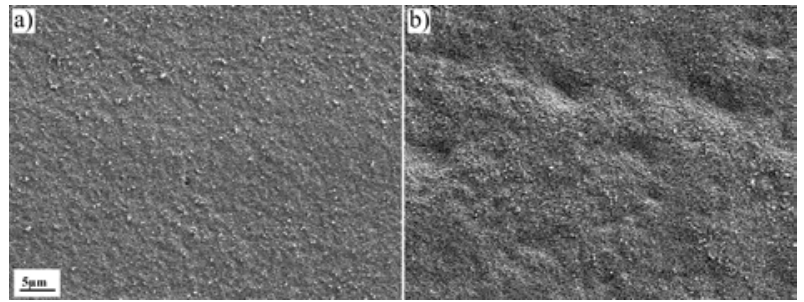

Fig. 11. SEM images (SE2 detector) of the surface quality for copper, $\lambda=532 \mathrm{~nm}, w_{0}=4.4 \mu \mathrm{m}, \phi_{0, s}=3.9 \mathrm{~J} / \mathrm{cm}^{2}$; a) Single pulse; b) 2 pulse-burst

If the material is changed to aluminum, the 2 pulse-burst lead to a smoother surface compared to single pulses for $1064 \mathrm{~nm}$ (see Fig. 12). Using a 2 pulse-burst suppresses the formation of small cavities and bumps on the surface. This smoothing of the surface was also observed for other materials [39]. But the melting worms on the surface are more pronounced for a 2 pulse-burst.

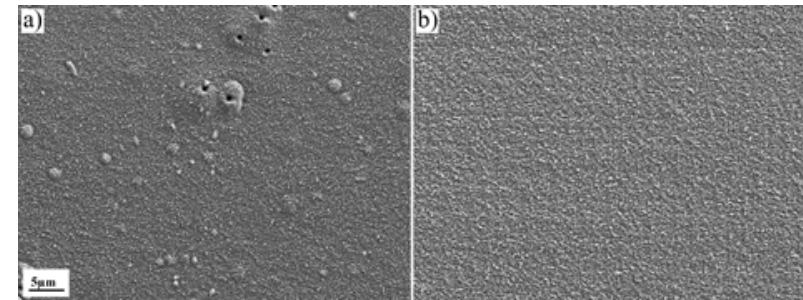

Fig. 12. SEM images (SE2 detector) of the surface quality for aluminum, $\lambda=1064 \mathrm{~nm}, w_{0}=15.5 \mu \mathrm{m}, \phi_{0, s}=1.6 \mathrm{~J} / \mathrm{cm}^{2}$;

a) Single pulse; b) 2 pulse-burst

The SEM images cannot finally confirm the assumption of a re-deposition of material with the second pulse. Especially for copper machined with $532 \mathrm{~nm}$ the observed surface quality gives no information regarding an eventual redeposition. The mechanism behind the negative contribution of the second pulse, i.e. the reduction of the maximum specific removal below $50 \%$ of the value observed for single pulses is still not fully understood. The structures are machined with several layers, meaning also multiple burstpackages. Therefore it may also be possible that the second pulse in the burst will influence the surface for the following burst package.

\subsection{3 pulse-burst}

For copper and aluminum using a spot radius of $15.5 \mu \mathrm{m}(1064 \mathrm{~nm})$ the 3 pulse-burst is more efficient than a 2 pulse-burst. If the second pulse of the 2 pulse-burst drives the particles in the plume back to the sample surface, the $3^{\text {rd }}$ pulse will no longer be shielded by any particles and impinges to the surface without any losses. This assumption is confirmed by the surfaces morphology of the structures observed using the SEM. The surfaces for a 3 pulseburst are similar to the surfaces observed with a single pulse for copper using $1064 \mathrm{~nm}$ (Fig. 13) and $532 \mathrm{~nm}$ (Fig. 14). The melting worms $(1064 \mathrm{~nm})$ or droplets $(532 \mathrm{~nm})$ are a bit larger for the 3 pulse-burst but in general the surfaces look similar to the ones observed with single pulses.

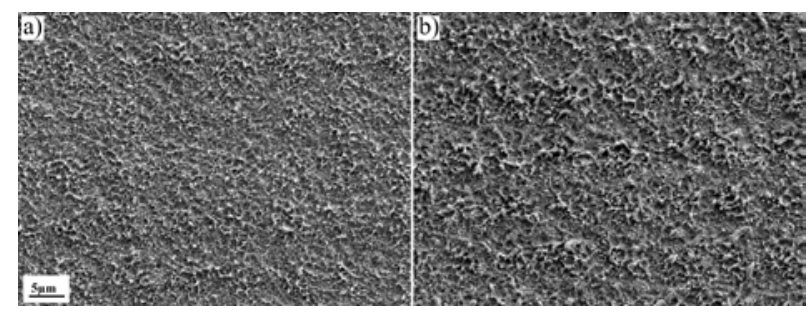

Fig. 13. SEM images (SE2 detector) of the surface quality for copper, $\lambda=1064 \mathrm{~nm}, w_{0}=15.5 \mu \mathrm{m}, \phi_{0, \mathrm{~s}}=2.2 \mathrm{~J} / \mathrm{cm}^{2}$; a) Single pulse; b) 3 pulse-burst

Also for aluminum the melting residues are less flat for the 3 pulse-burst compared to the 2 pulse-burst and larger than for single pulses.

Fig. 8 shows a much higher contribution to the ablation by the 3rd pulse compared to the 1st one. Therefore the surface must be pre-conditioned by the former pulses resulting in a higher contribution of the $3^{\text {rd }}$ pulse. These preconditioning could be a modification of the surface itself, e.g. the existence of surface structures or an additional heating of the surface by the back driven particles, which may be hot due to the absorption of the second pulse and may create a hot layer which influences the $3^{\text {rd }}$ pulse. In 
[40] that micro- and nanostructures on the surface strongly influence the absorption of the laser pulses. Therefore also the melting residues on the surface may play a role.

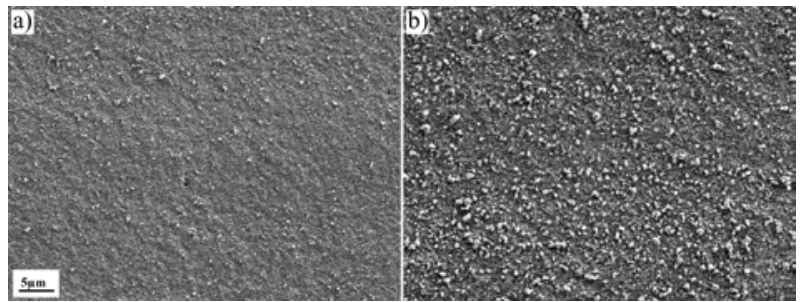

Fig. 14. SEM images (SE2 detector) of the surface quality for copper, $\lambda=532 \mathrm{~nm}, w_{0}=4.4 \mu \mathrm{m}, \phi_{0, s}=3.9 \mathrm{~J} / \mathrm{cm}^{2}$; a) Single pulse; b) 3 pulse-burst

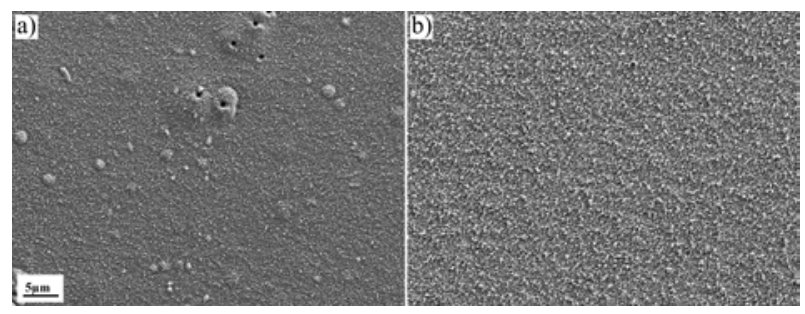

Fig. 15. SEM images (SE2 detector) of the surface quality for aluminum, $\lambda=1064 \mathrm{~nm}, w_{0}=15.5 \mu \mathrm{m}, \phi_{0, s}=1.6 \mathrm{~J} / \mathrm{cm}^{2}$;

a) Single pulse; b) 3 pulse-burst

Machining of stainless steel in contrast will not show this effect. The 3 pulse-burst is not more efficient than a 2 pulse-burst for both wavelengths. But also the energy penetration depth is much smaller for stainless steel, compared to the other tested materials, meaning less material is removed per pulse.

Machining copper and aluminum always show a higher specific removal rate for $532 \mathrm{~nm}$. Therefore the removed volume per area is higher in this case. If the specific removal rates of the pulse-burst experiments are now normed to the maximum rate observed with single pulses, then it can be shown, that the drop for the 2 pulse-burst is higher for $532 \mathrm{~nm}$ and the maximum rate of the 3 pulse-burst is significantly lower than the one for single pulses as illustrated in Fig. 10. This indicates a stronger shielding effect for $532 \mathrm{~nm}$ compared to $1064 \mathrm{~nm}$. This would be in line with the observed higher maximum specific removal rate for single pulses, as more material will be present in the particle plume, which is therefore more dense. But this behavior could also be explained by partially Rayleigh scattering in the plume, which would be significantly higher for shorter wavelengths. The exact mechanism behind these observations is still under investigations.

\subsection{4 to 8 pulse-burst}

For copper and using a spot radius of $15.5 \mu \mathrm{m}$ the specific removal rate again drops down if a 4 pulse-burst is used and exceeds the observed rate for single pulses using a 5 pulse-burst as shown in Fig. 1. We assume that the third pulse in the burst acts in a same way as the first pulse (impinges to the surface without any shielding) and the particle plume of the $3^{\text {rd }}$ pulse then again shields the $4^{\text {th }}$ pulse. The $5^{\text {th }}$ pulse then acts in a same way as the $3^{\text {rd }}$ pulse, in a 3 pulse-burst. The surfaces are covered by large melting worms which are larger for more pulses in the burst as illustrated in Fig. 16.

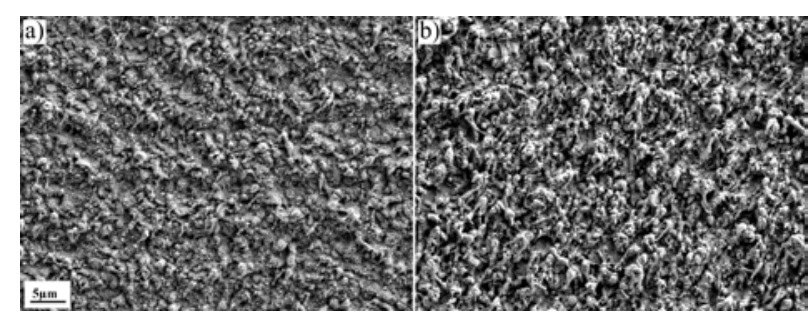

Fig. 16. SEM images (SE2 detector) of the surface quality for copper, $\lambda=1064 \mathrm{~nm}, w_{0}=15.5 \mu \mathrm{m}, \phi_{0, s}=2.2 \mathrm{~J} / \mathrm{cm}^{2}$; a) 4 pulse-burst;

b) 5 pulse-burst

The same tendency can also be observed for $532 \mathrm{~nm}$ wavelength (Fig. 17) with the restriction that neither the 3 pulse-burst nor the 5 pulse-burst exceeds the specific removal rate observed for a single pulse as shown in Fig. 3.

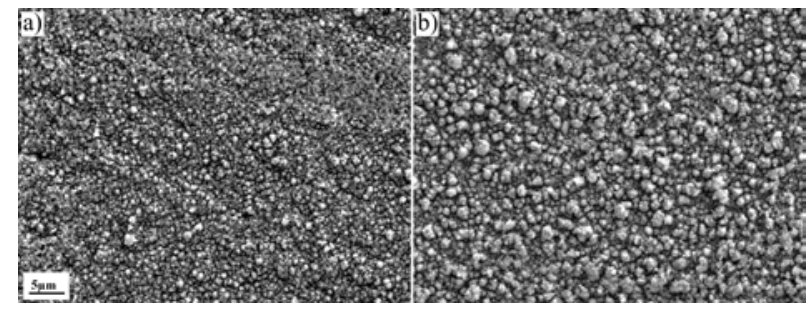

Fig. 17. SEM images (SE2 detector) of the surface quality for copper, $\lambda=532 \mathrm{~nm}, w_{0}=4.4 \mu \mathrm{m}, \phi_{0, s}=3.9 \mathrm{~J} / \mathrm{cm}^{2}$; a) 4 pulse-burst; b) 5 pulse-burst

\subsection{Influence of the spot size}

The influence of the spot size is investigated for both wavelengths and copper as sample material. In the case of IR irradiation (see Fig. 18) and using single pulses no difference in the specific removal rate can be observed for the two tested spot radii whereas for a 2 pulse-burst and a 3 pulse-burst a small influence is observed. For a 2 pulseburst the drop of the specific removal rate is a somewhat larger for the larger spot, i.e. $15.5 \mu \mathrm{m}$. The situation is appears to be completely different for a 3 pulse-burst, where the smaller spot radius $\left(w_{0}=11.2 \mu \mathrm{m}\right)$ shows a smaller specific removal rate compared to the spot radius of $15.5 \mu \mathrm{m}$. In addition for the smaller spot the maximum specific removal rate is no longer exceeding the rate observed for single pulses.

A change to $532 \mathrm{~nm}$ wavelength and smaller spots will result in a dependency of the specific removal rate on the spot radius already for single pulses, as shown in Fig. 19.

As shown in [41] copper shows two ablation regimes in the visible range measured for a spot radius of $20 \mu \mathrm{m}$. Therefore the specific removal rate can no longer be described by the simple model shown in [6]. The model to describe the specific removal rate has to be expanded to a two-threshold model according to [41]. Comparing the curves of the specific removal rates for the two different spot sizes shows a not negligible difference. Fitting the two-threshold model to the data points will result in the values for the threshold fluence and the energy penetrations depth summarized in Table 5. 


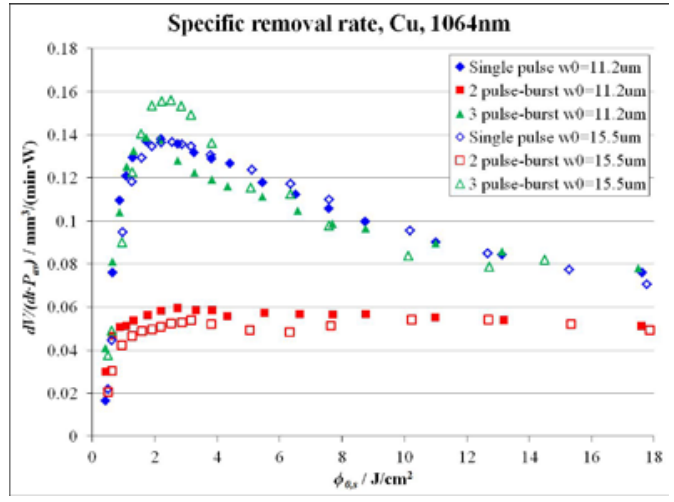

Fig. 18. Influence of the spot radius on the specific removal rate for copper, $\lambda=1064 \mathrm{~nm}$

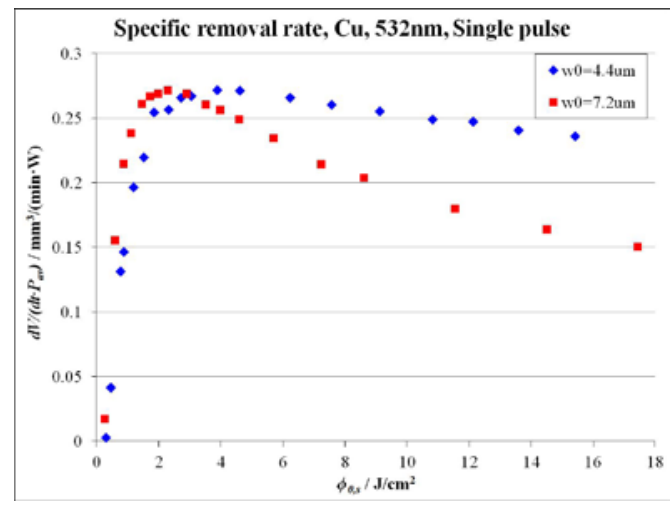

Fig. 19. Influence of the spot radius on the specific removal rate for copper, $\lambda=532 \mathrm{~nm}$ and single pulse operation

Table 5 Energy penetration depth and threshold fluence for the different regimes and spot sizes $(532 \mathrm{~nm})$

\begin{tabular}{cccc}
\hline & $w_{0}=20 \mu \mathrm{m}$ & $w_{0}=7.2 \mu \mathrm{m}$ & $w_{0}=4.4 \mu \mathrm{m}$ \\
\hline$\phi_{t h, 1} / \mathrm{J} / \mathrm{cm}^{2}$ & 0.11 & 0.072 & - \\
$\delta_{1} / \mathrm{nm}$ & 6.67 & 6.12 & - \\
$\phi_{t h, 2} / \mathrm{J} / \mathrm{cm}^{2}$ & 0.21 & 0.36 & 0.36 \\
$\delta_{2} / \mathrm{nm}$ & 32.78 & 56.21 & 55.4 \\
$\phi_{t h, 3} / \mathrm{J} / \mathrm{cm}^{2}$ & - & - & 1.15 \\
$\delta_{3} / \mathrm{nm}$ & - & - & 156.57 \\
\hline
\end{tabular}

The regime with the penetration depth of about $55 \mathrm{~nm}$ is observed for both spot sizes. For the radius of $7.2 \mu \mathrm{m}$ this regime is equal to the high ablation regime, where it is the low ablation regime for the spot radius of $4.4 \mu \mathrm{m}$. The regime with the lowest energy penetration depth contributes only a small amount to the ablation process. Therefore its influence can almost be neglected. This regime may also be present for the case with a spot radius of $4.4 \mu \mathrm{m}$, where two regimes with much higher ablation rates are found. Using the two-threshold model, only two regimes can be found and therefore the one with the lowest values may not be found. The presence of two ablation regimes is well known for fs-pulses as shown in [42]. In [43] it is also reported about the existence of a third regime for fs-pulses.

\section{Conclusions}

The specific removal rate for stainless steel, copper and aluminum was investigated regarding the influence of the burst mode using different wavelengths and spot sizes. It has found that the maximum specific removal rate for a 2 pulse-burst is always smaller than the maximum for single pulses. The maximum rate obtained by a 3 pulse-burst in the case of copper and aluminum is always higher than for a 2 pulse-burst and may exceed the maximum rate obtained with single pulses in special cases. In contrast, for stainless steel, the maximum rate of a 3 pulse-burst is smaller for $532 \mathrm{~nm}$ and $1064 \mathrm{~nm}$ respectively. The surface quality can likely be improved when pulse-bursts are applied. The appearance of small cavities and bumps can be shifted to higher fluences for steel and it can completely be avoided for aluminum machined at the optimum point.

There are many influencing factors, e.g. wavelength, spot radius, time spacing in the burst and number of pulses in the burst, which complicate the comparison of the results, also with other published observations. Additional investigations have to be performed for a better understanding of the ablation mechanism using pulse-bursts.

\section{Acknowledgments}

The authors thank Josef Zuercher for the help with the SEM micrographs Patrick Neuenschwander for the WLI measurements and Urs Hunziker for his work in the mechanical workshop. This work was supported by the European Union via the FP7 project APPOLO (GA 609355).

\section{References}

[1] C. Hoenninger, J. Akhil: Laser Technik Journal 13, (2016) 56.

[2] P. Russbueldt, T. Mans, J. Weitenberg, H. D. Hoffmann, R. Proprawe: Opt. Lett. 35, (2010) 4169.

[3] J.-P. Negel, A. Voss, M. Abdou Ahmed, D. Bauer, D. Sutter, A. Killi, T. Graf: Opt. Lett 38, (2013) 5442.

[4] C. Freitag, M. Wiedenmann, J.-P. Negel, A. Loescher, V. Onuseit, R. Weber, M. Abdou Ahmed, T. Graf: Appl. Phys. A 119, (2015) 1237.

[5] G. Raciukaitis, M. Brikas, P. Gecys, B. Voisiat, M. Gedvillas: J. Laser Micro/Nanoengin. 4, (2009) 186.

[6] B. Neuenschwander, G. F. Bucher, C. Nussbaum, B. Joss, M. Muralt, U. W. Hunziker, P. Schuetz: Proc. of SPIE 7584, (2010) 75840R.

[7] R. De Loor: Phys. Proc. 41, (2013) 544.

[8] S. Bruening, G. Hennig, S. Eifel, A. Gillner: Phys. Proc. 12, (2011) 105.

[9] B. Jaeggi, B. Neuenschwander, T. Meier, M. Zimmermann, G. Hennig: Phys. Proc. 41, (2013) 319.

[10] G. R. B. E. Roemer, P. Bechtold: Phys. Proc. 41, (2014) 29.

[11] O. Haupt, V. Schuetz, U. Stute: Proc. of SPIE 7921, (2011) 79210V.

[12] Z. Kuang, W. Perrie, J. Leach, M. Sharp, S. P. Edwardson, M. Padgett, G. Dearden, K. G. Watkins: Appl. Surf. Sc. 255, (2008) 2284.

[13]F. Zimmermann, S. Richter, S. Doering, A. Tuennermann, S. Nolte: Appl. Opt. 52, (2013) 1149.

[14]H. Zhang, S. M. Eaton, P. R. Hermann: Opt. Lett. 32, (2007) 2559.

[15] M. Kumkar, L. Bauer, S. Russ, M. Wendel, J. Kleiner, D. Grossmann, K. Bergner, S. Nolte: Proc. of SPIE 8972, (2014) 897214. 
[16] K. Mishchik, B. Chasagne, C. Javaux-Léger, C, Hoenninger, E. Mottay, R. Kling, J. Lopez: Proc. of SPIE 9740, (2016) 97400W.

[17]F. Hendricks, V. V. Matylitsky: Proc. of SPIE 9740, (2016) 97400Z.

[18] F. Hendricks, V. V. Matylitsky, M. Domke, H. P. Huber: Proc. of SPIE 9740, (2016) 97401A.

[19] R. Knappe, H. Haloui, A. Seifert, A. Weis, A. Nebel: Proc. of SPIE 7585, (2010) 75850H.

[20]B. Neuenschwander, T. Kramer, B. Lauer, B. Jaeggi: Proc. of SPIE 9350, (2015) 93500U.

[21]C. A. Hartmann, T. Fehr, M. Brajdic, A. Gillner: J. Laser Micro/Nanoengin. 2, (2007) 44.

[22] T. Kramer, Y. Zhang, S. Remund, B. Jaeggi, A. Michalowski, L. Grad, B. Neuenschwander: Proc. 17th Int. Symp. on Laser Precision Microfabrication, (2016).

[23] T. Kramer, B. Neuenschwander, B. Jaeggi, S. Remund, U. Hunziker, J. Zuercher: Phys. Proc. 83, (2016) 123.

[24] C. Kerse, HJ. Kalaycioglu, P. Elahi, B. Cetin, D. K. Kesimn, Ö. Akcaalan, S. Yavas, M. D. Asik, B. Öktem, H. Hoogland, R. Holzwarth, F. Ömer Ilday: Nature 537, (2016) 84.

[25] B. Lauer, B. Jaeggi, Y. Zhang, B. Neuenschwander: Proc. of ICALEO Paper M701, (2015).

[26] Y. Zhang, B. Lauer, B. Neuenschwander, V. Romano: Proc. of SPIE 9735, (2016) 97350M.

[27] O. Armbruster, A. Naghilou, M. Kitzler, W. Kautek: Appl. Surf. Sci. 396, (2017) 1736.

[28]B. Jaeggi, B. Neuenschwander, U. Hunziker, J. Zuercher, T. Meier, M. Zimmermann, K. H. Selbmann, G. Hennig: Proc. of SPIE 8243, (2012) 82430K.
[29] M. Zimmermann, B. Jaeggi, B. Neuenschwander: Proc. of SPIE 9350, (2015) 93500U.

[30] W. Hu, Y. C. Shin, G. King: Appl. Phys. A 98, (2010) 407.

[31] K. van der Straeten, F. Zibner, A. Olowinsky, A. Gillner: Proc. of ICALEO Paper 807, (2010).

[32] F. Bandi, N. Burdet, R. Carzino, A. Diaspro: Opt. Exp. 18, (2010) 23488.

[33] S. Amoruso, G. Ausanio, R. Bruzzese, L. Gragnaniello, L. Lanotte, M. Vitiello, X. Wang: Appl. Surf. Sci. 252, (2006) 4863.

[34] M. Sailer, F. Bauer, J. Kleiner, M. Kaiser: Lasers in Manufacturing Conference, (2015).

[35] A. Semerok, C. Dutouquet: Thin Film Solids 453-454, (2004) 501.

[36] M. E. Povarnitsyn, V. B. Fokin, P. Rl. Levashov, T. E. Itina: Phys. Rev. B 92, (2015) 174104.

[37] J. Koenig, S. Nolte, A. Tuennermann: Opt. Exp., 13, (2005) 10597.

[38]D. E. Roberts, A. du Plessis, L. R. Botha : Appl. Surf. Sci. 256, (2010) 1784.

[39]R. Mayerhofer : Proc. of SPIE 10091, (2017) 100910Z.

[40] A. Y. Vorobyev, C. Guo: Physica Rev. B 72, (2005) 195422.

[41]B. Jaeggi, B. Neuenschwander, S. Remund, T. Kramer: Proc. of SPIE 10091, (2017) 100910J.

[42] S. Nolte, C. Momma, H. Jacobs, A. Tünnermann, B. N. Chichkov, B. Wellegehausen, H. Welling: J. Opt. Soc. Am. B 14, (1997) 2716.

[43] M. Hashida, A. F. Semerok, O. Gobert, G. Petite, Y. Izawa, J. F. Wagner: Appl. Surf. Sci. 197-198, (2002) 862.

(Received: June 3, 2017, Accepted: November 20, 2017) 